\title{
Imaging Vulnerable Plaque in a Modern School of Cardiology - the Plaquelmage Project
}

\author{
Theodora Benedek
}

Editor-in-Chief, Journal of Interdisciplinary Medicine

\section{CORRESPONDENCE}

Thedora Benedek

Str. Gheorghe Marinescu nr. 38

540138 Târgu Mureș, Romania

Tel: +40 265215551

E-mail: theodora.benedek@gmail.com
Vulnerable plaques are atheromatous lesions with particular features that make them prone to rupture. Plaque rupture leads to the formation of an intracoronary thrombus, in most of the cases associated with an acute coronary syndrome.

The detection of vulnerable plaques is one of the main goals of modern cardiac imaging, and coronary computed tomography angiography remains the reference technique for plaque characterization due to its noninvasive nature associated with the possibility to provide complex information about plaque morphology and composition.

The PlaqueImage project started in 2015 with the aim to train a team of researchers in the modern imaging of coronary plaques, making them highly qualified in multimodality cardiac imaging. Along with the progress recorded in understanding the complex mechanisms related to plaque vulnerabilization and rupture, the PlaqueImage project also had the role to train $\mathrm{PhD}$ students in modern research methodology. As a result, $14 \mathrm{PhD}$ theses were completed, and a large number of publications on imaging vulnerable plaques were finalized. From the studies initiated by the PhD students, 8 have been published on the clinicaltrials.gov platform in order to validate them from a methodological point of view, and many study protocols of these trials have been published in the form of study designs. Some of them have been finalized, and some of them are still ongoing.

The ATHERODENT trial [NCT03395041] aimed to investigate the link between periodontal disease, inflammation, and atherosclerosis progression. ${ }^{1}$ The STAFF study investigated the association between in-stent flow hemodynamics and the risk of stent failure following bioresorbable vascular scaffold implantation. ${ }^{2}$ The CT-STENT study provides a cost-effectiveness perspective on provisional CT follow-up for MACE reduction after coronary stent implantation. The VIP trial [NCT03606330] investigated the association between systemic, pancoronary, and local plaque vulnerability for image-based prediction of acute coronary syndromes. ${ }^{3}$ The GEOMETRY study [NCT03702764] performed a computational assessment of plaque geometry in high-risk coronary lesions, ${ }^{4}$ while the STRESS study investigated the role of shear stress derived from im- 
aging-based computer modelling for the prediction of atheromatous plaque vulnerability. Hybrid 3D imaging has been developed by the HYBRIDHEART ${ }^{5}$ [NCT04397198] and FUSE-HEART ${ }^{6}$ [NCT04680689] studies, which used complex 3D CT/CMR hybrid imaging models for complex assessment of myocardial viability and ventricular contractility after myocardial infarction. Systemic inflammation as a major driver of major cardiac events has been tested in the VIABILITY study [NCT03830944] (aiming to study inflammatory and CMR-imaging based biomarkers for the assessment of ventricular remodeling and viability in the post-infarction period), ${ }^{7}$ the INFLAMAP study (aiming to identify inflammatory and imaging-based predictors of atrial fibrillation recurrence after pulmonary vein isolation), ${ }^{8}$ and the FIBROS study (aiming to identify imaging-derived biomarkers associated with atrial fibrosis, structural remodeling, and the risk of cardioembolic events). ${ }^{9}$ These are the main studies derived from the PlaqueImage project and coordinated by the research team of PlaqueImage.

The main results of PlaqueImage are also illustrated in the current issue of JIM. This focus issue presents the main features associated with plaque vulnerability in light of the principal results of the PlaqueImage project. For exemplification, spotty calcium, low-density atheroma, positive remodeling, and the napkin ring sign were identified as the most specific features associated with the risk of infarction. Also, serum biomarkers, such as matrix metalloproteases or inflammatory mediators, are associated with increased patient vulnerability, which adds significant risk on top of the plaque-related risk. Invasive cardiac imaging provides relevant information about vulnerability features as well, but its use is limited by the invasive nature of optical coherence tomography (OCT) and intravascular ultrasound (IVUS) techniques.

All these data have been extensively studied during the five years of PlaqueImage development, and the expertise accumulated during the project allowed the research team to identify the most relevant features associated with an increased risk of myocardial infarction, with a tremendous impact on public health and society. Altogether, the PlaqueImage project successfully demonstrated the role of a modern school of cardiology in the progress of science for the benefit of the patient.

\section{ACKNOWLEDGEMENT}

This research was supported via the research grant no. $103544 / 2016$, contract number 26/01.09.2016, entitled "Increasing the research capacity in the field of vulnerable plaque imaging, based on advanced nanoparticles, fusion imaging and computational simulation - PlaqueImage", financed by the Romanian Ministry of European Funds, the Romanian Government and the European Union.

\section{CONFLICT OF INTEREST}

Nothing to declare.

\section{REFERENCES}

1. Benedek T, Rodean I, Ratiu M, et al. Periodontal Disease, Inflammation and Atherosclerosis Progression in Patients with Acute Coronary Syndromes - the ATHERODENT Study. Journal of Cardiovascular Emergencies. 2018:4:17-23

2. Ferent I, Benedek I, Corduneanu A, Mester A, Benedek T, Benedek I. In-stent Flow Hemodynamics and the Risk of STent Failure Following Bioresorbable Vascular ScAFFolds Implantation - the STAFF Study. Journal of Interdisciplinary Medicine. 2017;2:320-323.

3. National Library of Medicine (U.S.) (2018 October - ). Systemic, Pancoronary and Local Coronary Vulnerability (VIP). Identifier NCT03606330. https:// www.clinicaltrials.gov/ct2/show/NCT03606330.

4. Ratiu M, Chitu M, Benedek I, et al. Impact of coronary plaque geometry on plaque vulnerability and its association with the risk of future cardiovascular events in patients with chest pain undergoing coronary computed tomographic angiography - the GEOMETRY study: Protocol for a prospective clinical trial. Medicine (Baltimore). 2018;97:e13498.

5. Stanescu A, Benedek I, Morariu M, Ratiu M, Zavate R, Mester A, Benedek T. New Imaging-derived Biomarkers Based on Tridimensional CTA/ MRI Hybrid Models for Complex Assessment of Myocardial Viability after Myocardial Infarction - the HYBRIDHEART Study. Journal of Interdisciplinary Medicine. 2018;3:16-20.

6. National Library of Medicine (U.S.) (2021 February -). Assessment of LesionAssociated Myocardial Ischemia Based on Fusion Coronary CT Imaging (FUSE-HEART). Identifier NCT04680689. https://www.clinicaltrials.gov/ ct2/show/NCT04680689.

7. Morariu M, Hodas R, Benedek T, et al. Impact of inflammation-mediated response on pan-coronary plaque vulnerability, myocardial viability and ventricular remodeling in the postinfarction period - the VIABILITY study: Protocol for a non-randomized prospective clinical study. Medicine (Baltimore). 2019;98:e15194.

8. Korodi S, Benedek T, Bordi L, et al. Inflammatory and Imaging-based Predictors of Atrial Fibrillation Recurrence after Pulmonary Vein Isolation Using Electroanatomical Mapping - the INFLAMAP Study. Journal of Interdisciplinary Medicine. 2018;3:10-15.

9. Korodi S, Toganel R, Benedek T, et al. Impact of inflammation-mediated myocardial fibrosis on the risk of recurrence after successful ablation of atrial fibrillation - the FIBRO-RISK study: Protocol for a non-randomized clinical trial. Medicine (Baltimore). 2019;98:e14504. 